A

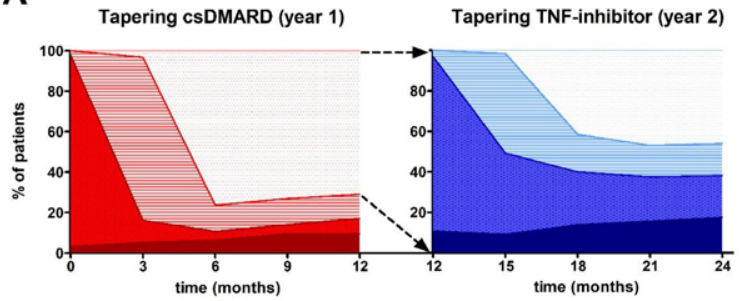

B

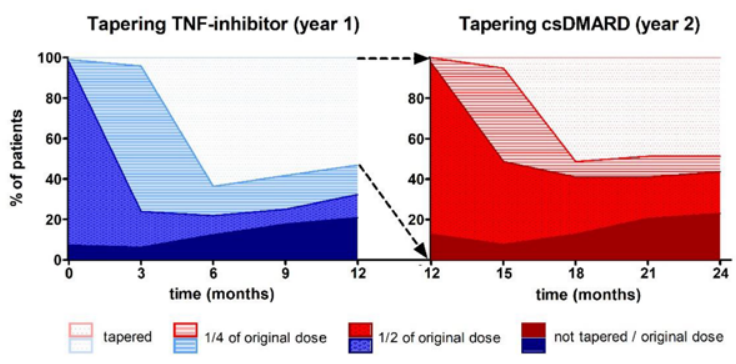

Figure 2 Overview of gradual tapering per time point.

Numbers indicate percentages of patients. Every 3 months the TNF-inhibitor or cSDMARD was tapered until after 6 months the drug could be stopped. In case of a flare, defined as DAS $>2.4$ and/or $S J C>1$, patients were set back at their previous effective dose. No further tapering was allowed after having a flare. For the second year (T12 - T24), only tapering status was shown for patients who - according to protocol - should have tapered their medication in the second year. csDMARD; conventional synthetic disease modifying antirheumatic drug.

Disclosure of Interests: None declared

DOI: 10.1136/annrheumdis-2020-eular.1417

\section{FRI0111 \\ TOCILIZUMAB MAY INDUCE SECONDARY HYPOGAMMAGLOBULINAEMIA. A RETROSPECTIVE CASE SERIES OF 42 PATIENTS}

F. Vílchez-Oya ${ }^{1}$, A. Pros ${ }^{1}$, I. Carrión Barberà ${ }^{1}$, J. A. Meraz Ostiz ${ }^{1}$, T. C. Salman Monte ${ }^{1}$, C. Perez-Garcia ${ }^{1} .{ }^{1}$ Consorci Parc de Salut Mar/Hospital del Mar, Rheumatology, Barcelona, Spain

Background: Tocilizumab (TCZ) is a recombinant humanized, anti-human monoclonal antibody of the immunoglobulin $\mathrm{G} 1_{\mathrm{k}}$ subclass directed against soluble and membrane-bound interleukin 6 receptors (IL-6R) [1].

Interleukin-6 (IL-6) has a pleiotropic effect on inflammation, immune response, and hematopoiesis. When it was first identified, it was named as B-cell-stimulating factor 2 (BSF-2) according to its ability to induce immunoglobulin production in Epstein-Barr virus-transformed B-cell lines or in Staphylococcus aureus Cowan 1-stimulated B cells [2-4].

Nowadays, it is known that IL-6 controls the survival, population expansion and maturation of B cells and plasmablasts. In that way, the regulation of Blimp-1 by STAT3 is linked to antibody secretion and is associated with long-lived plasma cells that produce large amounts of immunoglobulin. Furthermore, the ability of IL-6 to promote humoral immunity has been linked to its effects on follicular helper T cells where they promote B cell proliferation and immunoglobulin class switching [5].

Objectives: Hypogammaglobulinaemia is a known complication of some immunosuppressive drugs, not previously described in patients who received therapy with monoclonal antibody against the IL-6R. We aimed to analyzed the prevalence of hypogammaglobulinaemia in our series of patients treated with tocilizumab after a carefully diagnostic workup which ruled out other causes and analyzed whether is associated with a higher risk of infection

Methods: We conducted a retrospective review from 2010 to 2019 of forty-two patients affected with a rheumatic disease and treated with TCZ at our centre. In those patients in whom we had no record of immunoglobulin levels, we determined them in the blood analysis performed by usual clinical practice.

Results: 42 patients were identified, from whom 38 had rheumatoid arthritis. A $31 \%$ had immunoglobulin levels prior to starting treatment with TCZ but no one had hypogammaglobulinaemia. 2 patients were excluded due to their underlying disease could justify the IgG level abnormalities. During the treatment's follow-up, we identified that a $30 \%$ of the patients (12/40) had hypogammaglobulinaemia. Of those patients in whom immunoglobulin levels had been determined prior to starting treatment with TCZ, a $36.3 \%$ of them (4/11) developed hypogammaglobulinaemia during the follow-up. From the series, we observed a statistical significance tendency $(p=0.0057)$ for infection risk in those patients with hypogammaglobulinaemia in contrast to those with normal IgG level $(41.5 \%$ vs $14.3 \%$, respectively).

Conclusion: Secondary hypogammaglobulinaemia may occurs in patients receiving anti-IL6 agents such as tocilizumab and this could be associated with an increasing infection risk. The prevalence is not precisely known, in part because measurement of IgG prior to or during the treatment has not been a standard of care. No medical data have been previously disclosed about this possible adverse effect of anti-interleukin- 6 agents. Nevertheless, ideally randomized trials are needed to assess this initial hypothesis.

References:

[1] Sheppard M, Laskou F, Stapleton PP, Hadavi S, Dasgupta B. Tocilizumab (Actemra). Hum Vaccin Immunother. 2017;13(9):1972-1988.

[2] Tanaka T, Kishimoto T. The biology and medical implications of interleukin-6. Cancer Immunol Res. 2014;2(4):288-294.

[3] Tanaka T, Narazaki M, Kishimoto T. IL-6 in inflammation, immunity, and disease. Cold Spring Harb Perspect Biol. 2014;6(10):a016295. Published 2014 Sep 4.

[4] Kishimoto T. Interleukin-6: discovery of a pleiotropic cytokine. Arthritis Res Ther. 2006;8 Suppl 2(Suppl 2):S2.

[5] Hunter CA, Jones SA. IL-6 as a keystone cytokine in health and disease [published correction appears in Nat Immunol. 2017 Oct 18;18(11):1271]. Nat Immunol. 2015;16(5):448-457.

Disclosure of Interests: Francisco Vílchez-Oya: None declared, Ana Pros: None declared, Irene Carrión Barberà Grant/research support from: I received a grant from the Spanish Rheumatology Foundation (FER) and laboratories KERN PHARMA for a brief stay abroad., Juan Antonio Meraz Ostiz: None declared Tarek Carlos Salman Monte: None declared, Carolina Perez-Garcia: None declared

DOI: 10.1136/annrheumdis-2020-eular.2737

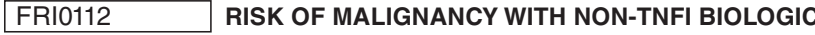 OR TOFACITINIB THERAPY IN RHEUMATOID ARTHRITIS: A META-ANALYSIS OF OBSERVATIONAL STUDIES}

W. Xie ${ }^{1}$, X. Yang ${ }^{1}$, Z. Zhang ${ }^{1} .{ }^{1}$ Peking University First Hospital, Department of Rheumatology and Clinical Immunology, Beijing, China

Background: With an increasing usage of non-TNFi biologics and tofacitinib, it is crucial to understand the comparative safety of these agents regarding malignancies risk.

Objectives: We aim to assess the risk of developing cancer in patients with RA exposed to non- TNF inhibitors (TNFi) biologics or tofacitinib therapy.

Methods: Systematical search of PubMed, EMBASE and Cochrane Library plus a hand search of conference proceedings were performed for observationa studies that reported cancer incidence in patients with RA treated with biologics or tofacitinib with active comparator of conventional DMARDs (csDMARDs) or TNFi. The pooled relative risk $(\mathrm{RR})$ and $95 \%$ confidence interval $(\mathrm{Cl})$ were calculated with fix-effects or random-effects model.

Results: Of 2,819 identified articles, a total of 10 studies involving over 323,361 patients and 1,179,263 patient-years of follow-up were included. Pooled analysis showed there was no increased risk of developing cancer in general or specific cancer types in RA patients receiving treatment with rituximab (pooled RR 1.13, $95 \% \mathrm{Cl} 0.80-1.59$ ), tocilizumab (pooled RR $0.96,95 \% \mathrm{Cl} 0.83-1.11$ ), or tofacitinib (pooled $\mathrm{RR} 0.97,95 \% \mathrm{Cl} 0.66-1.43$ ), compared with those receiving csDMARDs or TNFi. However, abatacept usage in RA was associated with a slightly increased overall cancer risk (pooled RR 1.13, 95\% Cl 1.02-1.24) and non-melanoma skin cancer (pooled RR 1.26, 95\% Cl 1.09-1.45), relative to csDMARDs or TNFi.

Conclusion: Compared with csDMARDs or TNFi, there was no increased risk of malignancies among RA patients treated with non-TNFi biologics or tofacitinib, with exception of abatacept associated with slightly increased total cancer and specific caner types. Extended researches are required to confirm the findings in a real-world context.

\section{References:}

[1] Smolen JS, Landewé R, Bijlsma J, et al. EULAR recommendations for the management of rheumatoid arthritis with synthetic and biological disease-modifying antirheumatic drugs: 2016 update. Ann Rheum Dis. 2017;76:960-977. 